\title{
Subchronic Glucocorticoid Receptor Inhibition Rescues Early Episodic Memory and Synaptic Plasticity Deficits in a Mouse Model of Alzheimer's Disease
}

\author{
Fabien Lanté1,2, Magda Chafai ${ }^{1,3}$, Elisabeth Fabienne Raymond',3, Ana Rita Salgueiro Pereira ${ }^{1,3}$, \\ Xavier Mouska', Scherazad Kootar', Jacques Barik', Ingrid Bethus' and Hélène Marie*,' \\ 'Institut de Pharmacologie Moléculaire et Cellulaire (IPMC), Centre National de la Recherche Scientifique (CNRS), Université de Nice Sophia \\ Antipolis, UMR 7275, Valbonne, France
}

\begin{abstract}
The early phase of Alzheimer's disease (AD) is characterized by hippocampus-dependent memory deficits and impaired synaptic plasticity. Increasing evidence suggests that stress and dysregulation of the hypothalamo-pituitary-adrenal (HPA) axis, marked by the elevated circulating glucocorticoids, are risk factors for AD onset. How these changes contribute to early hippocampal dysfunction remains unclear. Using an elaborated version of the object recognition task, we carefully monitored alterations in key components of episodic memory, the first type of memory altered in AD patients, in early symptomatic Tg2576 AD mice. We also combined biochemical and ex vivo electrophysiological analyses to reveal novel cellular and molecular dysregulations underpinning the onset of the pathology. We show that HPA axis, circadian rhythm, and feedback mechanisms, as well as episodic memory, are compromised in this early symptomatic phase, reminiscent of human AD pathology. The cognitive decline could be rescued by subchronic in vivo treatment with RU486, a glucocorticoid receptor antagonist. These observed phenotypes were paralleled by a specific enhancement of N-Methyl-D-aspartic acid receptor (NMDAR)-dependent LTD in CAI pyramidal neurons, whereas LTP and metabotropic glutamate receptor-dependent LTD remain unchanged. NMDAR transmission was also enhanced. Finally, we show that, as for the behavioral deficit, RU486 treatment rescues this abnormal synaptic phenotype. These preclinical results define glucocorticoid signaling as a contributing factor to both episodic memory loss and early synaptic failure in this AD mouse model, and suggest that glucocorticoid receptor targeting strategies could be beneficial to delay $A D$ onset.

Neuropsychopharmacology (20I5) 40, I772-I78I; doi:I0.I038/npp.20I5.25; published online I8 February 20I5
\end{abstract}

\section{INTRODUCTION}

Alzheimer's disease (AD) is the most common cause of dementia in aging human populations, but only low-efficacy palliative treatments are currently available. The hippocampus and the type of memory it encodes (ie episodic memory) are affected very early in all forms of $\mathrm{AD}$ (deToledo-Morrell et al, 2007; Salmon and Bondi, 2009). Since the discovery of $\mathrm{A} \beta$ as the main constituent of senile plaques observed in $\mathrm{AD}$ patient brains and the cloning of the amyloid precursor protein (APP), there has been an intense effort to understand how APP misprocessing and $\mathrm{A} \beta$ affect hippocampal function.

* Correspondence: Dr H Marie, Institut de Pharmacologie Moléculaire et Cellulaire (IPMC), Centre National de la Recherche Scientifique (CNRS), Université de Nice Sophia Antipolis, UMR 7275, 660 Route des Lucioles, 06560 Valbonne, France, Tel: +33 4939534 40, Fax: +33 4939534 08, E-mail: marie@ipmc.cnrs.fr

${ }^{2}$ Present address: Grenoble Institut des Neurosciences, INSERM U836, Université Joseph Fourier, Equipe 12 Neuropathologies et Dysfonctions Synaptiques, 38706 La Tronche Cedex, France

${ }^{3}$ These authors equally contributed to this work.

Received 16 May 2014; revised 13 January 2015; accepted I5 January 2015; accepted article preview online 27 January 2015
There is now a strong evidence that $\mathrm{A} \beta$ accumulation into soluble oligomers in this structure is most probably one of the main triggers of the pathology leading to early synaptic failure and subsequent memory loss (Palop and Mucke, 2010; Shankar et al, 2008). However, the exact cellular mechanism contributing to the onset of synaptic failure and memory loss in $\mathrm{AD}$ remains unclear.

Preclinical and clinical data suggest that stress is an important environmental risk factor leading to $\mathrm{AD}$ (Rothman and Mattson, 2010). Indeed, patients with a high level of distress proneness are 2.7 times more likely to develop $\mathrm{AD}$, and this trait is also associated with a faster progression of the disease (Wilson et al, 2003, 2007). This is in line with the observations that major stressful events lower the age of onset of familial AD (Mejia et al, 2003). In various preclinical $\mathrm{AD}$ models, stress worsens deficits in hippocampus-dependent spatial learning (Catania et al, 2009; Cuadrado-Tejedor et al, 2012; Dong et al, 2004; Jeong et al, 2006; Srivareerat et al, 2009; Tran et al, 2010). These deleterious effects are in part caused by an accelerated accumulation of $\mathrm{A} \beta$ under stressing conditions (Catania et al, 2009; Cuadrado-Tejedor et al, 2012; Dong et al, 2004; Green et al, 2006; Jeong et al, 2006; Srivareerat 
et al, 2009). Stress triggers the release of glucocorticoid hormones (CORT), the major output of the hypothalamopituitary-adrenal axis (HPA). CORT acts via two related receptors, the mineralocorticoid (MR) and the glucocorticoid receptors (GR). GR has a low affinity for CORT and is thus believed to be a key player under elevated CORT levels, although a role of MR cannot be excluded. Both AD patients and $\mathrm{AD}$ mouse models display a dysregulated HPA axis, marked by a mild hypercortisolemia that is apparent early during the pathology (Csernansky et al, 2006; Elgh et al, 2006; Hebda-Bauer et al, 2013; Nasman et al, 1995; Rasmuson et al, 2001; Weiner et al, 1997). Increased CORT levels are sufficient to exacerbate $\mathrm{A} \beta$ deposits, and a recent study suggested that antagonism of GR could prevent this effect (Baglietto-Vargas et al, 2013). Albeit this increasing evidence for a synergistic relationship between dysregulated HPA function and $\mathrm{AD}$ pathogenesis, its cellular underpinnings remain poorly understood.

Using chronic $\mathrm{A} \beta$ infusion in adult rat brains, the group of Alkadhi suggested a relationship between $\mathrm{A} \beta$, in vivo synaptic plasticity and chronic psychosocial stress, showing that this type of stress exacerbates $\mathrm{A} \beta$-induced alterations in hippocampal long-term potentiation (LTP) and long-term depression (LTD; Tran et al, 2011a, 2011b). These data argue for a role of stress signaling in $\mathrm{AD}$-related synaptic dysfunction, but there are currently no other reports addressing this point in more detail. Importantly, there is paucity of data regarding the contribution of CORT signaling to $\mathrm{AD}$-related hippocampal synapse failure ie, weakening of synaptic communication, a phenotype which is believed to drive the onset of memory loss in AD as we and others have shown previously (D'Amelio et al, 2011; Selkoe, 2002). Identifying how a dysregulation of CORT signaling contributes to synaptic failure in $\mathrm{AD}$ is of importance as CORT has a prominent role in shaping hippocampal synaptic plasticity (Chaouloff and Groc, 2011).

Here, we used the Tg2576 transgenic AD mouse model, which displays $\mathrm{AD}$-like $\mathrm{A} \beta$ accumulation as oligomers with late formation of plaques in memory-encoding areas (hippocampus and neocortex) during aging (Hsiao et al, 1996). We previously demonstrated that onset of cognitive deficits in this model correlated with low levels of synaptic AMPA receptors and a stronger LTD of AMPAR responses in hippocampal CA1 neurons (D'Amelio et al, 2011). We now further characterize these memory and synaptic deficits in the early symptomatic phase of this model and provide strong evidence for a role of dysregulated HPA activity to these deficits. Our results define enhanced CORT signaling, via GRs, as a contributing factor to both early synaptic failure and episodic memory loss in this mouse model.

\section{MATERIALS AND METHODS}

\section{Mice}

Hemizygous Tg2576 mice carrying the human Swedish mutation (hAPPswe transgenene: human APP695 with double mutations at KM670/671NL) are hybrids between C57BL6 and SLJ provided by Taconic Biosciences (D'Amelio et al, 2011; Hsiao et al, 1996). Tg2576 and wild-type (WT) littermates were used for all experiments. The original transgenic mouse was developed using B6SJLF2 zygotes
(Hsiao et al, 1996). The mouse is a hybrid between C57BL6 and SLJ (Hsiao et al, 1996) and back-crossed to C57BL/6 mice for two generations (Carlson et al, 1997) before being deposited at Taconic Biosciences. Hemizygous males were back-crossed with $\mathrm{C} 57 \mathrm{BL} / 6 \mathrm{NTac}$ for derivation at Taconic Biosciences and the colony is maintained by mating hemizygous male mice with B6SJL/F1 female mice (Taconic Model 1349). These hemizygous mice are not congenic or co-isogenic mice, as they contain mixed genetic backgrounds of C57BL/6 and SJL mice. The hemizygous and WT littermates may be different in alleles near the transgene site because the original mouse and breeder mice were backcrossed to $\mathrm{C} 57 \mathrm{BL} / 6$ mice. Thus we cannot rule out the possibility that the phenotypic difference between $\mathrm{Tg}$ and WT mice could be because of the allelic difference instead of the transgene (Wolfer et al, 2002). Four-month old Tg2576 mice were used, except for in Figures $1 \mathrm{c}$ and $3 \mathrm{e}$ where 1-month old mice were used. For behavioral, plasma corticosterone levels, dexamethasone suppression test, and RU486 experiments, only male mice were used (see Supplementary Materials and Methods for additional details on animal care).

\section{Plasma CORT Measurements and Dexamethasone Suppression Test}

To determine plasma CORT levels, blood was collected from the submandibular vein without anesthesia (see Supplementary Materials and Methods for details). For circadian plasma CORT measurements, blood was collected at the onset of the light resting phase (0800 hours) and the dark active phase (2000 hours) in the same animal. After 15 min of centrifugation at $2000 \times g$, at $4{ }^{\circ} \mathrm{C}$, plasma samples were stored at $-80^{\circ} \mathrm{C}$. Plasma corticosterone concentrations were measured using an Enzyme Immunoassay (EIA) kit following the manufacturer's instructions (Enzo Life Science, France). For the dexamethasone suppression test, dexamethasone 21-phosphate disodium salt $(0,05 \mathrm{mg} / \mathrm{kg}$; SigmaAldrich, France) dissolved in saline $(\mathrm{NaCl}, 0.9 \%)$ or vehicle (saline) were injected intraperitoneally in WT and Tg2576 mice at 1200 hours. Blood was collected at 1800 hours and plasma corticosterone levels were assessed using the EIA kit.

\section{RU486 Treatment}

RU486 (40 mg/kg) was dissolved in DMSO for electrophysiology experiments or in water containing a droplet of Tween-20 for behavioral experiments and injected twice daily subcutaneously in the interscapular region of mice 2 days or 4 days before they were killed. The appropriate vehicle solution $\left(\mathrm{H}_{2} \mathrm{O} /\right.$ Tween-20) was administered accordingly. For ex vivo electrophysiological experiments, RU486 $(0.5 \mu \mathrm{M})$ or DMSO (vehicle) was directly applied to the slices via the bath perfusion throughout the recording.

\section{Object Recognition Paradigm}

The episodic-like object recognition protocol was performed essentially as described previously (Dere et al, 2005) with minor variations in the protocol to minimize stress levels (see Supplementary Materials and Methods for 

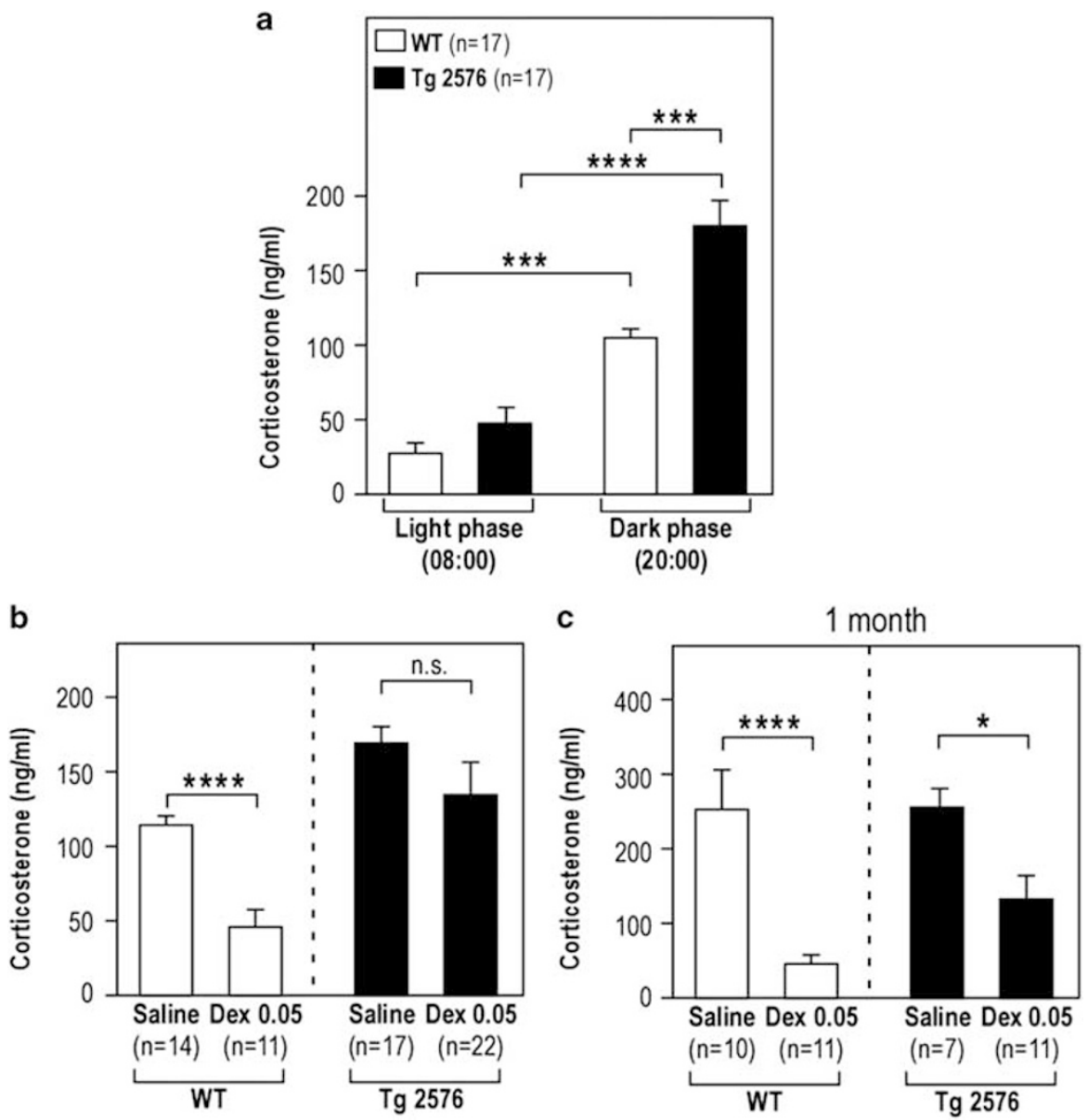

Figure I Four-month old Tg2576 mice display alterations in corticosterone signaling (a) Plasma corticosterone levels (ng/ml) were measured by ELISA at the onset of the light/resting (0800 hours) and dark/awake (2000 hours) phases in 4-month old male WT (white bars) and Tg2576 (black bars) mice. Tg2576 mice display significantly elevated levels of corticosterone at the onset of the dark phase compared with WT. (b and c) Plasma corticosterone levels $(\mathrm{ng} / \mathrm{ml})$ were measured by ELISA 6 hours after in vivo injection of dexamethasone (Dex) at 0,05 mg/kg or saline (b) in 4-month old WT (white) and Tg2576 (black) mice; (c) in I-month old WT (white) and Tg2576 (black) mice. One-month old WT and Tg2576 mice, and 4-month old WT mice display the expected decrease in corticosterone levels after Dex injection, whereas 4-month old Tg2576 mice do not. $* p<0.05$; $* * * *<0.00$ I, ***** $p<0.000$ I, n.s., non-significant. $n=$ number of mice. See Supplementary Table I for the statistics details.

details). This task is based on the paradigm that episodic memory is the memory of personal experiences and specific events including what happened ('What' component), location ('Where' component) and time ('When' component). A schematic description of the procedure is shown in Figure 2a. Using these test trial exploration data, we estimated three components of episodic-like memory: What $=($ exploration time $(\mathrm{ET})$ 'olds' $-\mathrm{ET}$ 'recents' $) /(\mathrm{ET}$ 'olds' + ET 'recents'); When $=(\mathrm{ET}$ 'old stationary' $-\mathrm{ET}$ 'recents')/(ET 'old stationary' + ET 'recents'); Where $=(\mathrm{ET}$ 'old displaced' - ET 'old stationary')/(ET 'old displaced' + ET 'old stationary'; see Supplementary Materials and Methods for details).

\section{Electrophysiology}

Hippocampal slices were prepared as described previously (see Supplementary Materials and Methods for details). Field excitatory post-synaptic potentials (fEPSPs) were recorded in the stratum radiatum of the CA1 region and stimuli delivered to the Schaeffer Collateral Pathway by a monopolar glass electrode filled with ACSF (see Supplementary Materials and Methods for details). LTP was induced using a TBS protocol: 10 bursts at $5 \mathrm{~Hz}$ repeated 10 times in $15 \mathrm{~s}$ intervals. Each burst consisted of four pulses of $100 \mathrm{~Hz}$ (see Supplementary Materials and Methods for details of analysis procedure). LTD was induced with a low frequency stimulation (LFS) protocol of 900 pulses at $1 \mathrm{~Hz}$. To induce NMDAR-dependent LTD, slices were superfused with standard ACSF containing NMDA $(20 \mu \mathrm{M})$ for $2.5 \mathrm{~min}$. To induce mGluR-dependent LTD, slices were superfused with standard ACSF containing DHPG ((S)-3,5-Dihydroxyphenylglycine; $100 \mu \mathrm{M}$ or $50 \mu \mathrm{M})$ for $15 \mathrm{~min}$ in the presence of the NMDAR antagonist D,LAPV ((2R)-amino-5-phosphonovaleric acid, $100 \mu \mathrm{M})$ throughout the recordings. Whole-cell voltage-clamp recordings were made from CA1 pyramidal cells (see Supplementary Materials and Methods for details). NMDA-evoked currents were recorded with an $\mathrm{Mg}^{2+}$-free ASCF containing DNQX $(20 \mu \mathrm{M})$ and picrotoxin $(50 \mu \mathrm{M})$ at different holding potentials from $-60 \mathrm{mV}$ to $+40 \mathrm{mV}$ with $20 \mathrm{mV}$ step increments. An average of current over ten 
a
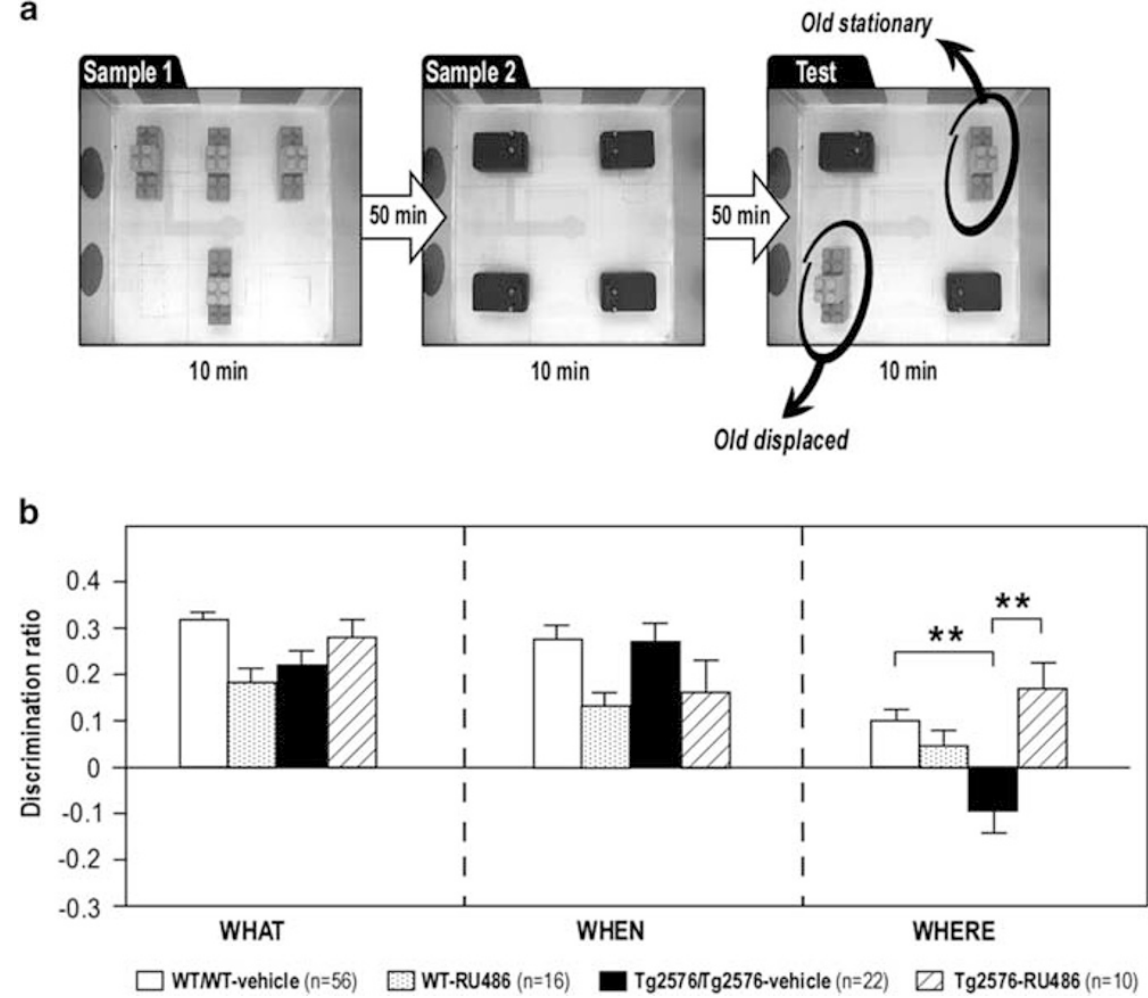

Figure 2 Four-month old Tg2576 mice display episodic memory deficits, a behavioral phenotype which is rescued by blocking glucocorticoid receptors. (a) Schema of the What-When-Where object recognition protocol. (b) Episodic-like memory in 4-month old untreated/vehicle-treated WT (white bars), in 4-day in vivo RU486-treated WT (dotted bars), in untreated/vehicle-treated Tg2576 (black bars) and in 4-day in vivo RU486-treated Tg2576 (hashed black bars) mice. Discrimination ratios for each component of episodic-like memory are indicated. $* * *<0.0$ I; $n=$ number of mice. See Supplementary Table I for the statistics details.

sweeps was calculated per holding potential, which was then normalized to the value obtained at $-60 \mathrm{mV}$ (representing -1 on graphs). The data were pooled by condition and represented as mean \pm SEM. Data analysis was performed with the clampfit software (Molecular devices).

\section{Statistical Analysis}

For Dex tests and electrophysiological recordings, statistics were performed with an unpaired two-tailed Student's $t$-test to probe for a difference between genotype (WT, Tg2576). A twoway analysis of variance (ANOVA) was used to test for an effect of treatment (vehicle, RU486) or genotype (WT, Tg2576) on behavior and electrophysiology data and repeated measures two-way ANOVAs were used to test for an effect of phase of the day (light phase, dark phase) or genotype (WT, Tg2576) on corticosterone measurements. Post hoc analysis was conducted with Bonferroni's comparison. All the statistical analyses were done with using Prism 6 (Graph Pad). All numerical data are presented as mean \pm SEM. $p<0.05$ was considered to be statistically significant. See Supplementary Data for full statistics Supplementary Tables 1, 2, and 3.

\section{RESULTS}

\section{Four-Month Old Tg2576 Mice Display Alterations in HPA Axis Function}

In many species, plasma CORT levels are subjected to circadian rhythmicity that is key for maintaining normal homeostasis. Dysregulation of this pattern of secretion has been described in several disease states (Lightman and Conway-Campbell, 2010). Therefore, we first investigated the integrity of the HPA axis in 4-month old Tg2576 mice and WT littermates. We assayed nadir CORT plasma levels at the onset of the light phase ( 0800 hours, resting period for rodents) and peak levels at the onset of the dark phase (2000 hours, active period for rodents). Both genotypes displayed the expected circadian elevation in CORT levels at the onset of the dark phase (Figure 1a, Supplementary Table 1). We observed that, although morning CORT levels did not significantly differ between the two genotypes, Tg2576 mice displayed significantly higher levels of plasma CORT in the evening at the onset of the active phase (Figure 1a, Supplementary Table 1). These data indicate that a clear HPA axis dysregulation occurs at an early stage of AD pathogenesis in Tg2576 mouse model. To get further insights into this dysregulation, we sought to determine if there were modifications in the feedback loops that regulate CORT secretion. Thus, we submitted the 4-month old Tg2576 mice and WT littermates to the commonly employed dexamethasone (Dex) suppression test. Injection of this potent selective glucocorticoid receptor agonist triggers a negative feedback mechanism resulting in a reduction in plasma CORT levels within hours. As expected, we observed a marked reduction in plasma CORT levels in WT mice 6 hours after Dex injection when compared with the saline-injected controls (Figure 1b, Supplementary Table 1). In striking contrast, Dex did not 
significantly alter CORT levels in Tg2576 mice (Figure $1 \mathrm{~b}$, Supplementary Table 1), suggesting pronounced disruption of the GR-dependent feedback mechanism of HPA axis regulation. We then examined the age dependency of this dysregulation in 1-month old mice. At this age, the Dex suppression test was normal in Tg2576 mice (Figure 1c, Supplementary Table 1), consistent with observations that, at this age, no cognitive deficits have been evidenced and little to no $\mathrm{A} \beta$ accumulation is present in these mutant mice (D'Amelio et al, 2011; Hsiao et al, 1996; Mustafiz et al, 2011). This suggests that HPA axis deregulation is owing to chronic misprocessing of APP and accumulation of APPderived peptides and not because of the over-expression of hAPPswe per se. Together, these data demonstrate that, at 4 months of age, the homeostasis of the HPA axis is compromised in these transgenic mice, leading to chronically elevated levels of circulating CORT.

\section{Four-Month Old Tg2576 Mice Display Episodic Memory Deficits, which can be Reversed by Glucocorticoid Receptor Antagonism}

To assess AD-related cognitive decline, we evaluated episodic memory in 4-month old WT and Tg2576 mice. Previous analyses of cognitive function in these mice at this age focused on other types of hippocampus-dependent memory tasks, such as the Morris water maze or contextual fear conditioning (D'Amelio et al, 2011; Stewart et al, 2011). These tasks, however, do not test for episodic memory, which is the first type of memory that is affected in $A D$ patients (deToledo-Morrell et al, 2007; Salmon and Bondi, 2009). To specifically assess this type of memory in our mice, we used an elaborated version of the object recognition (OR) task, which can probe for the 'What', 'When', and 'Where' components of episodic memory (Dere et al, 2005). Exploration time for objects and discrimination ratio were evaluated during the test trial (Figure 2a). We observed that mice from both genotypes display a similar total exploration time during the $10 \mathrm{~min}$ test trial $(\mathrm{WT}=63.56 \pm 5.73 \mathrm{~s} ; \mathrm{Tg} 2576=59.62 \pm 6.11 \mathrm{~s} ; p>0.05$, data not shown). These data argue against any alterations in general locomotion or anxiety-like behavior in Tg2576 mice at this age, which could have compromised the outcome of this behavioral test. We calculated the discrimination ratios for the 'What', 'When', and 'Where' components of episodic memory. For the 'What' and 'When' components, the positive discrimination ratio for the two genotypes did not differ (see Figure $2 \mathrm{~b}$ and Supplementary Table 1 ). However, for the 'Where' component, although WT control animals (untreated/vehicle-treated mice) displayed a positive discrimination ratio, $\mathrm{Tg} 2576$ mice (untreated/vehicle-treated) displayed a marked negative discrimination ratio, significantly different from WT Figure $2 \mathrm{~b}$ and Supplementary Table 1). These data demonstrate that, while WT mice displayed a good episodic memory in this refined object recognition paradigm, the Tg2576 mice failed to process the 'Where' component of this task, which represents in itself the full integration of the episodic memory (object, time, and location).

In light of these data and the clear deregulation of the HPA axis, we next examined whether there was a correlation between excess CORT signaling and memory deficits. To reach this goal, we hypothesized that blocking GRs could rescue this memory deficit, as these receptors are abundant in the hippocampus. It is indeed reasonable to speculate that, as CORT is chronically elevated, the GRs became overactive in the Tg2576 mice. WT or Tg2576 mice received twice daily subchronic (4 days) injections of the commonly employed GR antagonist, RU486, before being assessed in the episodic memory paradigm. Figure $2 \mathrm{~b}$ reports discrimination ratios for the 'What', 'When', and 'Where' components of episodic memory in RU486-treated mice compared with the control mice (untreated/vehicle treated; see also Supplementary Table 1). Pharmacological blockade of GRs by RU486 treatment did not significantly alter episodic memory in WT, although a trend towards lower memory for the 'What' and 'When' components was apparent (Figure 2b, Supplementary Table 1). RU486, however, fully rescued the deficit in the 'Where' component of episodic memory in Tg2576 mice, as RU486-treated Tg2576 mice performed, as well as WT mice (Figure 2b, Supplementary Table 1). Together these data suggests that abnormally-elevated CORT levels trigger GRs over-activation, which in turn impairs episodic memory formation in these early symptomatic mice.

NMDAR-Dependent LTD is Abnormally Enhanced in 4Month Old Tg2576 Mice, whereas mGluR-Dependent LTD and LTP Remain Intact

To further characterize the cellular underpinnings of this memory deficit, we investigated synaptic plasticity in 4month old WT and Tg2576 mice. We performed field electrophysiology in the CA1 area of ex vivo hippocampal slices. Schaeffer collaterals were stimulated to evoke fEPSPs at the CA3-CA1 synapses. Using this technique, we previously reported that electrically induced LTP of the AMPAR response remained intact, whereas LTD was enhanced in the 3-month old Tg2576 mice (D'Amelio et al, 2011). We now confirm these phenotypes at 4 months of age (Figure $3 a$ and $b$ ).

CA1 AMPAR LTD can be induced by activation of NMDA receptors (NMDARs) or by activation of group I metabotropic glutamate receptors (mGluRs; Luscher and Huber, 2010; Malenka and Bear, 2004). We therefore next asked which type of LTD is primarily affected in Tg2576 mice at this age, by recording NMDA-induced and DHPG-induced chemical LTD, to assess NMDARs- and mGluRs-dependent LTD, respectively. NMDA application generated an NMDAR-dependent LTD in both genotypes, but this LTD was significantly exacerbated in Tg2576 mice when compared with the WT mice (Figure 3c). In contrast, mGluR-dependent LTD, induced by the application of $100 \mu \mathrm{M}$ of DHPG, was similar in both genotypes (Figure 3d). This lack of difference in Tg2576 mice was not owing to saturation of the mGluR-dependent LTD as use of lower DHPG concentrations $(50 \mu \mathrm{M})$ resulted in similar LTD magnitudes in WT and Tg2576 mice (data not shown-WT: $59.68 \pm 7.3 \%$; Tg2576: $65.34 \pm 3.92 \%, p<0.05$ ).

To correlate this specific NMDAR-dependent LTD with chronic misprocessing of APP and accumulation of APPderived peptides and not to over-expression of hAPPswe per se, we recorded NMDA-induced LTD in 1-month old mice. At this age, Tg2576 and WT mice exhibit comparable 

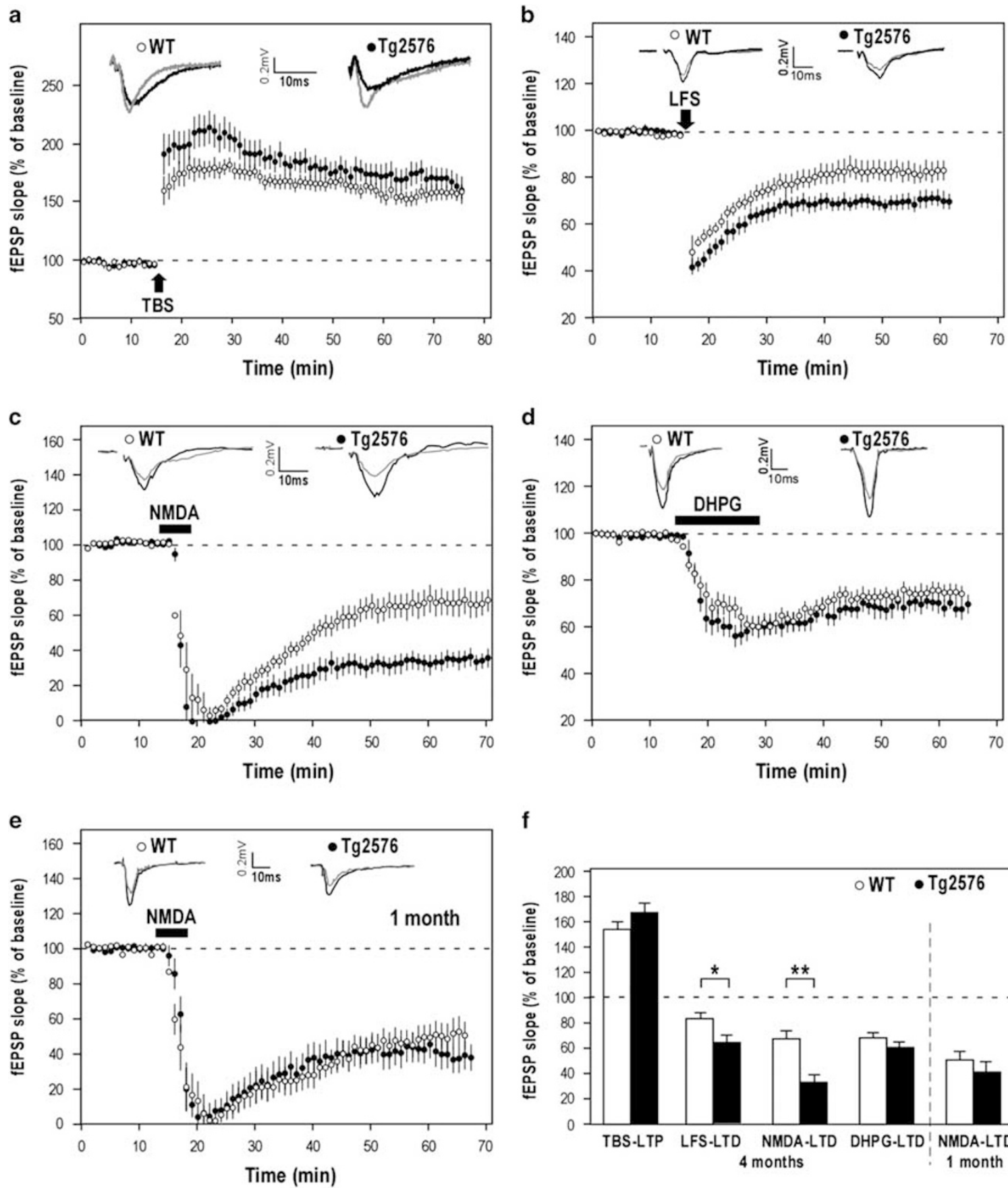

f

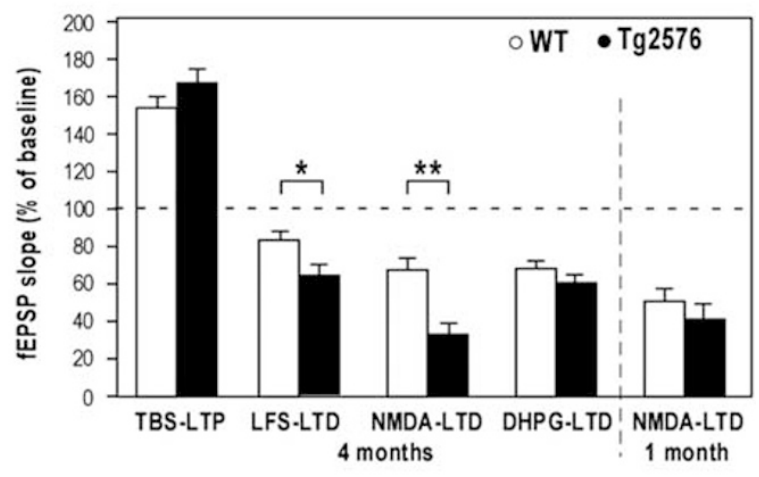

Figure 3 NMDAR-dependent LTD is enhanced at the CA3-CAI synapse in 4-month old Tg2576 mice compared with the 4-month old WT littermates, whereas mGLuR-dependent LTD and LTP remain intact. Traces in a-e show sample fEPSPs pre- (black) and post- (gray) LTP or LTD induction. (a) Summary graphs of electrically induced LTP (theta burst stimulation (TBS), arrow) in 4-month old WT $(n=3$ mice, $N=8$ slices) and Tg2576 ( $n=3, N=9$ ) mice as percentage of fEPSP baseline. (b) Summary graphs of electrically induced LTD (low frequency stimulation (LFS), arrow) displayed in 4-month old WT $(n=5, N=8)$ and Tg2576 ( $n=4, N=8)$ mice. (c) Summary graphs of NMDAR-dependent LTD (induced by the application of NMDA $20 \mu M / 2.5$ min) displayed in 4-month old WT $(n=4, N=10)$ and Tg2576 $(n=4, N=9)$ mice. (d) Summary graphs of mGluR-dependent LTD (induced by the application of DHPG $100 \mu \mathrm{M} / 15 \mathrm{~min})$ displayed in 4-month old WT $(n=4, N=10)$ and Tg2576 $(n=4, N=12)$ mice. (e) Summary graphs of NMDAR-dependent LTD (induced by application of NMDA $20 \mu \mathrm{M} / 2.5 \mathrm{~min}$ ) displayed in I-month old WT $(n=4, N=12)$ and Tg2576 $(n=4, N=12)$ mice. ( $f$ ) Average LTP or LTD magnitudes calculated from graphs in a-e in last 10 min of recordings (as percentage of baseline). ${ }^{*} p<0,05$; ** $p<0,0$ I. See Supplementary Table 2 for the statistics details.

NMDA-induced LTD (Figure 3e). A summary graph of these electrophysiology data is presented in Figure 3f. Together, these results demonstrate that the episodic memory deficit observed in the 4-month old Tg2576 mice correlates with a pathologically enhanced NMDAR-dependent LTD in CA1 pyramidal neurons. 


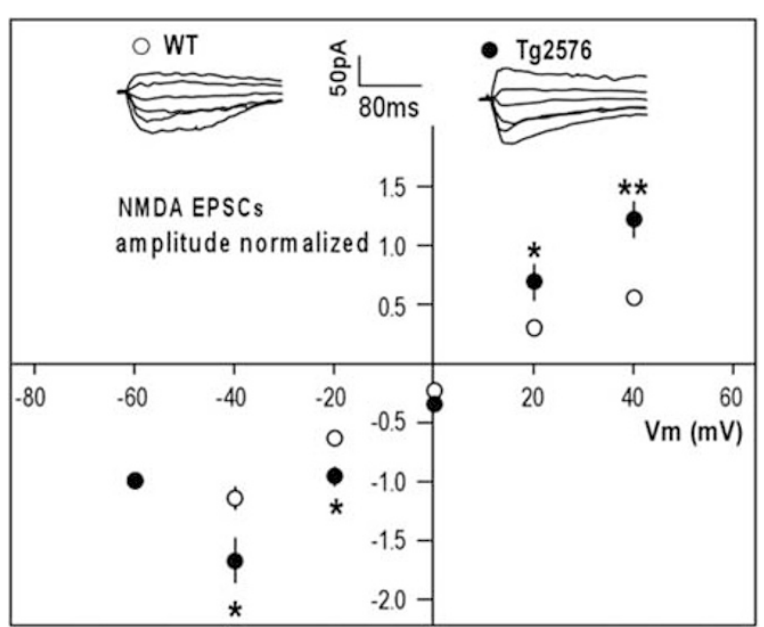

Figure 4 NMDAR currents are higher in CAI of 4-month old Tg2576 mice compared with the WT. Current (I, normalized to NMDA EPSC amplitude at $-60 \mathrm{mV})$ to Voltage $(\mathrm{Vm})$ relationships of NMDA currents in CAI pyramidal neurons in 4-month old WT ( $n=7$ mice, $N=10 / 8$ cells) and Tg2576 $(n=5, N=8)$ mice. Sample EPSCs are shown at $V m$ from $-60 \mathrm{mV}$ to $+40 \mathrm{mV}$. $* p<0,05$; $* * * 0,0$ I. See Supplementary Table 2 for the statistics details.

\section{Four-Month Old Tg2576 Mice Display Abnormally High NMDAR Currents}

NMDAR-dependent LTD requires activation of synaptic NMDARs. To determine whether this transmission could be perturbed in 4-month old Tg2576 mice, we analyzed NMDAR currents of CA1 pyramidal neurons. We measured the current-voltage relationship of evoked synaptic NMDARmediated excitatory post-synaptic currents (EPSCs) in CA1 pyramidal neurons by whole-cell voltage clamp recordings. The amplitude of evoked NMDAR EPSCs was significantly enhanced in Tg2576 mice compared with that of WT at the various membrane potentials $(\mathrm{Vm})$ tested (Figure 4).

\section{Four Days of in vivo RU486 Treatment is Necessary to Reverse the NMDAR-dependent LTD Phenotype in 4-Month Old Tg2576 Mice}

In light of the reversal of episodic memory defect observed in Tg2576 mice following subchronic RU486 treatment (Figure 2), we next tested whether this therapeutic effect resulted in the normalization of excessive NMDAR-dependent LTD, hence providing a cellular basis for this evident behavioral phenotype. Indeed, we observed that the LTD induced by NMDA application was significantly reduced in RU486-treated Tg2576 mice compared to vehicle-treated Tg2576 mice (Figure 5a), returning to WT values (see summary graph in Figure $5 \mathrm{c}$ and Supplementary Table 3 for statistical analysis). Vehicle- and RU486-treated WT mice exhibited identical pattern of NMDA-induced LTD (Supplementary Figure S1A). These data suggest that the dose of RU486 employed is sufficient to normalize excessive CORT signaling in CA1 neurons of Tg2576 mice, without altering the physiological LTD process as observed in WT mice.

To address the time dependency required for this cellular rescue, we tested whether direct in vitro application or shorter (two days) in vivo administration of RU486 were effective. None of these two treatments could reverse the pathologically enhanced LTD observed in Tg2576 mice (Supplementary Figure S2; see also summary graph in Figure $5 \mathrm{c}$ ). These data demonstrate that several days of RU486 treatment is necessary to successfully reverse this synaptic plasticity alteration.

\section{Electrically Induced LTD and NMDAR Currents are Normalized by 4 Days of in vivo RU486 Treatment in Tg2576 Mice}

We also tested the rescue potential of this 4-day in vivo RU486 treatment on electrically induced LTD. As for NMDA-induced LTD, the enhanced LFS-induced LTD we observed in Tg2576 mice (Figure $3 \mathrm{~b}$ ) was rescued by this treatment (Figure 5b), returning to WT levels (Figure 5c and Supplementary Table 3 for statistical analysis). Again, RU486 administration did not significantly alter LFSinduced LTD in WT mice (Supplementary Figure S1B). A summary graph of the rescue experiments attempted on the LTD phenotypes in Tg2576 mice and in WT littermates is provided in Figure $5 \mathrm{c}$.

Finally, we tested if this 4-day in vivo RU486 treatment could normalize the excess of NMDAR current exhibited by Tg2576 mice (Figure 4). Whole-cell patch clamp recordings of CA1 pyramidal neurons from vehicle-treated and RU486treated Tg2576 mice demonstrated that RU486 administration could significantly reduce NMDAR currents (Figure 5d), bringing them back to WT levels (Figure 5d: dashed line).

\section{DISCUSSION}

In recent years, numerous studies examined the relationship between CORT signaling and AD pathology, especially $\mathrm{A} \beta$, with noticeable emphasis on behavioral and biochemical aspects of the disease. The major finding of this work is a precise pharmacological dissection of the changes in synaptic plasticity that occur at an early stage of the pathology with strong evidence for a central role of CORT in this process. We had previously reported normal LTP and enhanced LTD in CA1 neurons in this early symptomatic phase (D'Amelio et al, 2011). We now demonstrate that this enhanced synaptic depression selectively depends on NMDAR transmission, whereas mGluR contribution remains spared. Using whole-cell patch clamp recordings, we could attribute this phenotype to enhanced NMDAR EPSCs. These results are in agreement with the role played by NMDARs in this type of LTD (Malenka and Bear, 2004). Importantly, we demonstrate that the synaptic alterations are rescued by subchronic in vivo administration of the GR antagonist, RU486.

In vivo stress protocols, as well as in vitro and in vivo CORT application in rodents, mainly by recruiting GRs, which are abundant in the hippocampus, were shown to enhance both CA1 NMDAR- and mGluR-dependent LTD (Chaouloff and Groc, 2011). These protocols were also shown to either increase or decrease CA1 LTP depending on the CORT doses tested or the type of stress studied (acute or chronic; Chaouloff and Groc, 2011). Yet, only NMDARdependent LTD is affected in our model. It is therefore 
a

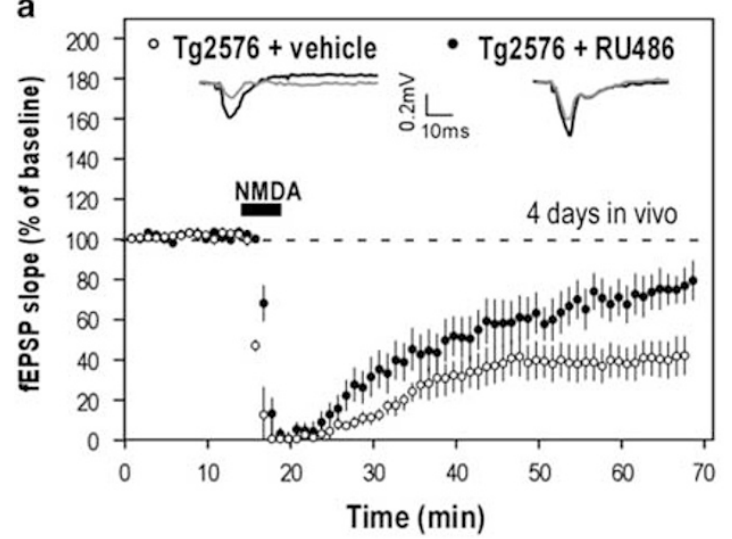

C

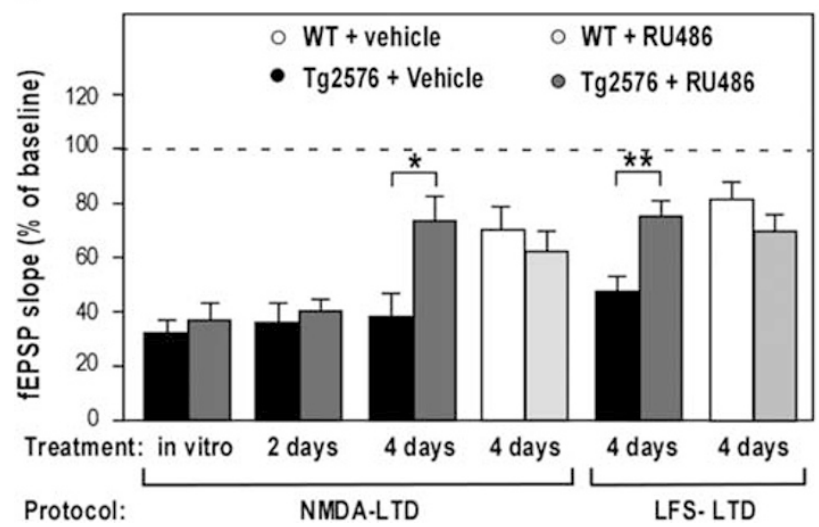

b

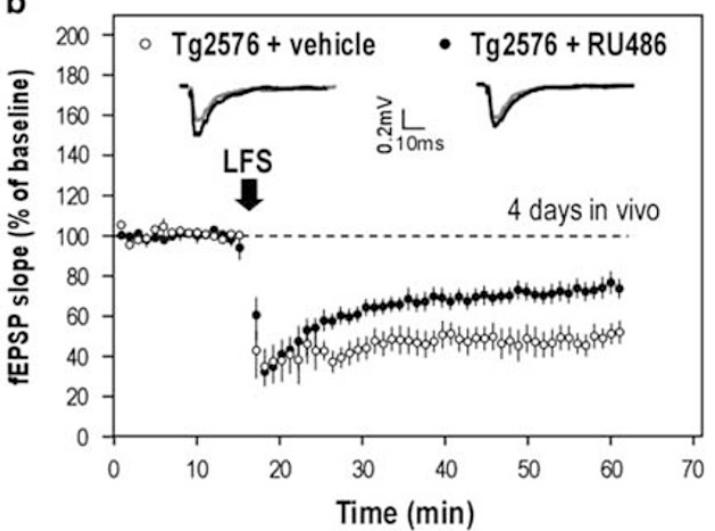

d

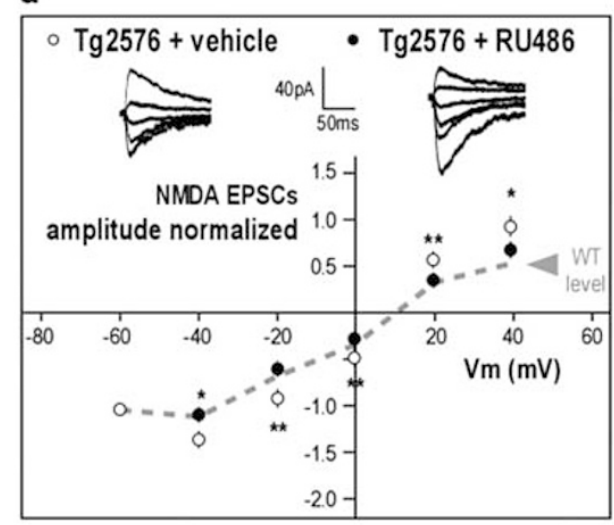

Figure 5 Four days of in vivo RU486 treatment reverses the LTD and NMDAR phenotypes at the CA3-CAI synapse in 4-month old Tg2576 mice. Summary graphs (as percentage of fEPSP baseline) of a NMDAR-dependent LTD (induced by application of NMDA $20 \mu M / 2.5$ min) after 4 days of vehicle ( $n=4$ mice, $N=8$ slices) or RU486 ( $n=4, N=10)$ treatment; (b) electrically induced LTD (low frequency stimulation (LFS), arrow) after 4 days of vehicle ( $n=4$ mice, $N=7$ slices) or RU486 ( $n=5, N=1 \mathrm{I}$ ) treatment; Traces in a and b show sample fEPSPs pre- (black) and post- (gray) LTD induction. (c) Average LTD magnitudes calculated from graphs in $5 \mathrm{a}$ and $5 \mathrm{~b}$, and Supplementary Figures I and 2 in last 10 min of recordings (as percentage of baseline). (d) NMDAR currents are normalized by four days of in vivo RU486 treatment in Tg2576 mice. Current (I, normalized to NMDAR EPSC amplitude at - 60 mV) to Voltage $(\mathrm{Vm})$ relationships of NMDAR currents in CAI pyramidal neurons in 4-month old Tg2576 mice after 4 days of vehicle $(n=4$ mice, $N=9$ cells) or RU486 ( $n=4$ mice, $N=1$ I cells) treatment. Dashed line represents WT IN relationship of Figure 4. Sample EPSCs are shown at Vm from $-60 \mathrm{mV}$ to $+40 \mathrm{mV}$. ${ }^{*} p<0,05 ; * * * 0,01$. Student's t-test statistical results are shown with the asterisks. See Supplementary Table 3 for the statistics details and two-way ANOVAs statistics.

unlikely that the AD-related synaptic plasticity phenotype is simply because of the elevated CORT, but more probably owing to an intricate synergy between chronic APP misprocessing and increasing CORT levels, to which NMDAR-dependent LTD is particularly sensitive. The fact that we could fully reverse this aberrant synaptic phenotype with 4 days, and not 2 days, in vivo RU486 treatment supports the idea that GR-dependent genomic effects must be necessary to reset a homeostatic balance of CORT signaling in Tg2576 mice. In line with this idea, altering GR expression has been shown to modulate the hippocampal expression of several glutamate receptors and to trigger cognitive dysfunction (Wei et al, 2007), reinforcing the link between CORT/GR, glutamatergic plasticity and altered behaviors. We cannot at present exclude additional implication of the progesterone receptor in the rescue of the synaptic phenotype since this receptor is also blocked by this compound (Baulieu, 1997). This issue will in the future benefit from the generation of double GR KO/Tg2576 mice. Nevertheless, other recently published data strongly argue for a specific GR-mediated beneficial effect of RU486 on the rescue of behavioral and biochemical phenotypes in another $\mathrm{AD}$ mouse model with more advanced neurodegeneration (Baglietto-Vargas et al, 2013).

In a previous publication, we had reported a caspase- and calcineurin-dependent pathological mechanism leading to lower levels of synaptic AMPAR in the hippocampus of these early symptomatic mice (D'Amelio et al, 2011). As both caspase and calcineurin have been implicated in hippocampal NMDAR-dependent LTD (Li et al, 2010b; Mulkey et al, 1994), they could also contribute to the enhanced LTD we further studied here. It will be important, in future studies, to identify the interplay between this previously identified pathological mechanism and the novel CORT-dependent mechanism we report now. These two cellular pathways are certainly not mutually exclusive and could in fact be intimately linked as a study in $\mathrm{AD}$ and several studies in immunology research suggest (Distelhorst, 2002; Li et al, 2010a).

Copious information is available on the mnemonic deficits displayed by aged animal models of AD (Ashe and Zahs, 2010; Stewart et al, 2011). Here, we provide solid 
evidence for an early impairment in episodic memory in 4month old Tg2576 mice, correlating with early aggregation of $\mathrm{A} \beta$ into oligomers, but before plaque formation (Hsiao et al, 1996; Mustafiz et al, 2011). To our knowledge, this is the first report of perturbation in this type of memory in the early phase of $\mathrm{AD}$ in $\mathrm{AD}$ mice, as few other studies tested this mnemonic deficit at more advanced stages of the pathology (Baglietto-Vargas et al, 2013; Davis et al, 2013; Good et al, 2007). In light of the impaired HPA axis feedback and the key role played by CORT in facilitating the consolidation of emotionally-charged memories (McGaugh and Roozendaal, 2002), it was crucial to monitor episodic memories in minimized stress conditions as presented here. We can therefore attribute the observed memory deficit to a prolonged dysregulation of the HPA axis accompanied by APP misprocessing and not to acute changes in CORT levels induced by the design of the behavioral paradigm. A 4 days subchronic antagonism of GR was sufficient to have fully beneficial outcomes on memory impairment, corroborating a recent study in older $3 x$ Tg mice with more advanced $A D$ pathology, which employed a chronic infusion of GR antagonist for 3 weeks (Baglietto-Vargas et al, 2013). This shorter daily treatment holds the advantage of minimizing the possible side effects of prolonged GR blockade (Morgan and Laufgraben, 2013).

As described in other preclinical and clinical studies (Csernansky et al, 2006; Hebda-Bauer et al, 2013; Nasman et al, 1995; Rasmuson et al, 2001), we report an altered circadian rhythm of the HPA axis early in our mouse model. Whether this results from a dysregulation of the pulsatile ultradian pattern of CORT secretion is however yet to be demonstrated. Ultradian hormone pulsatility is inherent within the HPA axis as a result of negative feedback (Walker et al, 2010). The lack of responding of 4-month old Tg2576 mice to the Dex suppression test clearly argues for a strong impairment of this loop owing to the altered APP processing, hence indicating that ultradian rhythm may also be compromised. The Dex suppression test was normal in 1-month old mice, suggesting that the dysregulation occurs slowly, may be depending on $\mathrm{A} \beta$ accumulation, before senile plaque and without tau alterations (Hsiao et al, 1996; Mustafiz et al, 2011).

Although, the presented data and the work from other labs now provide convincing behavioral, biochemical, and electrophysiological evidence that CORT and $\mathrm{A} \beta$ are intimately linked (Rothman and Mattson, 2010), which one of CORT or $\mathrm{A} \beta$ accumulation occurs first in our model remains to be fully elucidated. Nevertheless, our data demonstrate that RU486 treatment readily reverses episodic memory and pathologically enhanced LTD present in the early symptomatic phase in our mouse model, thus strongly arguing for a pathological synergistic interaction between chronic APP misprocessing and HPA axis dysregulation.

\section{FUNDING AND DISCLOSURE}

This work was financed by the ATIP/AVENIR program (Centre national de la recherche scientifique-CNRS) to HM; by the French Fondation pour la Coopération ScientifiquePlan Alzheimer 2008-2012 (Senior Innovative Grant 2010) to FL, MC, EFR and HM; by the French Government (National Research Agency, ANR) through the 'Investments for the Future' LABEX SIGNALIFE: program reference \#ANR-11-LABX-0028-01 to HM and an Erasmus student grant to ARSP. The authors declare no conflict of interest.

\section{ACKNOWLEDGEMENTS}

We thank Dr Beurrier (IBDML-CNRS, Marseille, France) for critical reading of the manuscript.

\section{REFERENCES}

Ashe KH, Zahs KR (2010). Probing the biology of Alzheimer's disease in mice. Neuron 66: 631-645.

Baglietto-Vargas D, Medeiros R, Martinez-Coria H, LaFerla FM, Green KN (2013). Mifepristone alters amyloid precursor protein processing to preclude amyloid beta and also reduces tau pathology. Biol Psychiatry 74: 357-366.

Baulieu EE (1997). RU 486 (mifepristone). A short overview of its mechanisms of action and clinical uses at the end of 1996. Ann NY Acad Sci 828: 47-58.

Carlson GA, Borchelt DR, Dake A, Turner S, Danielson V, Coffin JD et al (1997). Genetic modification of the phenotypes produced by amyloid precursor protein overexpression in transgenic mice. Hum Mol Genet 6: 1951-1959.

Catania C, Sotiropoulos I, Silva R, Onofri C, Breen KC, Sousa N et al (2009). The amyloidogenic potential and behavioral correlates of stress. Mol Psychiatry 14: 95-105.

Chaouloff F, Groc L (2011). Temporal modulation of hippocampal excitatory transmission by corticosteroids and stress. Front Neuroendocrinol 32: 25-42.

Csernansky JG, Dong H, Fagan AM, Wang L, Xiong C, Holtzman DM et al (2006). Plasma cortisol and progression of dementia in subjects with Alzheimer-type dementia. Am J Psychiatry 163: 2164-2169.

Cuadrado-Tejedor M, Ricobaraza A, Frechilla D, Franco R, Perez-Mediavilla A, Garcia-Osta A (2012). Chronic mild stress accelerates the onset and progression of the Alzheimer's disease phenotype in Tg2576 mice. J Alzheimers Dis 28: 567-578.

D'Amelio M, Cavallucci V, Middei S, Marchetti C, Pacioni S, Ferri A et al (2011). Caspase-3 triggers early synaptic dysfunction in a mouse model of Alzheimer's disease. Nat Neurosci 14: 69-76.

Davis KE, Easton A, Eacott MJ, Gigg J (2013). Episodic-like memory for what-where-which occasion is selectively impaired in the 3xTgAD mouse model of Alzheimer's disease. J Alzheimers Dis 33: 681-698.

Dere E, Huston JP, De Souza Silva MA (2005). Episodic-like memory in mice: simultaneous assessment of object, place and temporal order memory. Brain Res Brain Res Protoc 16: 10-19.

deToledo-Morrell L, Stoub TR, Wang C (2007). Hippocampal atrophy and disconnection in incipient and mild Alzheimer's disease. Prog Brain Res 163: 741-753.

Distelhorst CW (2002). Recent insights into the mechanism of glucocorticosteroid-induced apoptosis. Cell Death Differ 9: 6-19.

Dong H, Goico B, Martin M, Csernansky CA, Bertchume A, Csernansky JG (2004). Modulation of hippocampal cell proliferation, memory, and amyloid plaque deposition in APPsw (Tg2576) mutant mice by isolation stress. Neuroscience 127: 601-609.

Elgh E, Lindqvist AA, Fagerlund M, Eriksson S, Olsson T, Nasman B (2006). Cognitive dysfunction, hippocampal atrophy and glucocorticoid feedback in Alzheimer's disease. Biol Psychiatry 59: 155-161.

Good MA, Hale G, Staal V (2007). Impaired "episodic-like" object memory in adult APPswe transgenic mice. Behav Neurosci 121: $443-448$. 
Green KN, Billings LM, Roozendaal B, McGaugh JL, LaFerla FM (2006). Glucocorticoids increase amyloid-beta and tau pathology in a mouse model of Alzheimer's disease. J Neurosci 26: 9047-9056.

Hebda-Bauer EK, Simmons TA, Sugg A, Ural E, Stewart JA, Beals JL et al (2013). 3xTg-AD mice exhibit an activated central stress axis during early-stage pathology. J Alzheimers Dis 33: 407-422.

Hsiao K, Chapman P, Nilsen S, Eckman C, Harigaya Y, Younkin S et al (1996). Correlative memory deficits, Abeta elevation, and amyloid plaques in transgenic mice. Science 274: 99-102.

Jeong YH, Park CH, Yoo J, Shin KY, Ahn SM, Kim HS et al (2006). Chronic stress accelerates learning and memory impairments and increases amyloid deposition in APPV717I-CT100 transgenic mice, an Alzheimer's disease model. FASEB J 20: 729-731.

Li WZ, Li WP, Yao YY, Zhang W, Yin YY, Wu GC et al (2010a). Glucocorticoids increase impairments in learning and memory due to elevated amyloid precursor protein expression and neuronal apoptosis in 12-month old mice. Eur J Pharmacol 628: $108-115$.

Li Z, Jo J, Jia JM, Lo SC, Whitcomb DJ, Jiao S et al (2010b). Caspase-3 activation via mitochondria is required for long-term depression and AMPA receptor internalization. Cell 141: 859-871.

Lightman SL, Conway-Campbell BL (2010). The crucial role of pulsatile activity of the HPA axis for continuous dynamic equilibration. Nat Rev Neurosci 11: 710-718.

Luscher C, Huber KM (2010). Group 1 mGluR-dependent synaptic long-term depression: mechanisms and implications for circuitry and disease. Neuron 65: 445-459.

Malenka RC, Bear MF (2004). LTP and LTD: an embarrassment of riches. Neuron 44: 5-21.

McGaugh JL, Roozendaal B (2002). Role of adrenal stress hormones in forming lasting memories in the brain. Curr Opin Neurobiol 12: 205-210.

Mejia S, Giraldo M, Pineda D, Ardila A, Lopera F (2003). Nongenetic factors as modifiers of the age of onset of familial Alzheimer's disease. Int Psychogeriatr 15: 337-349.

Morgan FH, Laufgraben MJ (2013). Mifepristone for management of Cushing's syndrome. Pharmacotherapy 33: 319-329.

Mulkey RM, Endo S, Shenolikar S, Malenka RC (1994). Involvement of a calcineurin/inhibitor-1 phosphatase cascade in hippocampal long-term depression. Nature 369: 486-488.

Mustafiz T, Portelius E, Gustavsson MK, Holtta M, Zetterberg H, Blennow $\mathrm{K}$ et al (2011). Characterization of the brain betaamyloid isoform pattern at different ages of $\mathrm{Tg} 2576$ mice. Neurodegener Dis 8: 352-363.

Nasman B, Olsson T, Viitanen M, Carlstrom K (1995). A subtle disturbance in the feedback regulation of the hypothalamicpituitary-adrenal axis in the early phase of Alzheimer's disease. Psychoneuroendocrinology 20: 211-220.

Palop JJ, Mucke L (2010). Amyloid-beta-induced neuronal dysfunction in Alzheimer's disease: from synapses toward neural networks. Nat Neurosci 13: 812-818.
Rasmuson S, Andrew R, Nasman B, Seckl JR, Walker BR, Olsson T (2001). Increased glucocorticoid production and altered cortisol metabolism in women with mild to moderate Alzheimer's disease. Biol Psychiatry 49: 547-552.

Rothman SM, Mattson MP (2010). Adverse stress, hippocampal networks, and Alzheimer's disease. Neuromolecular Med 12: 56-70.

Salmon DP, Bondi MW (2009). Neuropsychological assessment of dementia. Annu Rev Psychol 60: 257-282.

Selkoe DJ (2002). Alzheimer's disease is a synaptic failure. Science 298: 789-791.

Shankar GM, Li S, Mehta TH, Garcia-Munoz A, Shepardson NE, Smith I et al (2008). Amyloid-beta protein dimers isolated directly from Alzheimer's brains impair synaptic plasticity and memory. Nat Med 14: 837-842.

Srivareerat M, Tran TT, Alzoubi KH, Alkadhi KA (2009). Chronic psychosocial stress exacerbates impairment of cognition and long-term potentiation in beta-amyloid rat model of Alzheimer's disease. Biol Psychiatry 65: 918-926.

Stewart S, Cacucci F, Lever C (2011). Which memory task for my mouse? A systematic review of spatial memory performance in the Tg2576 Alzheimer's mouse model. J Alzheimers Dis 26: 105-126.

Tran TT, Srivareerat M, Alhaider IA, Alkadhi KA (2011a). Chronic psychosocial stress enhances long-term depression in a subthreshold amyloid-beta rat model of Alzheimer's disease. J Neurochem 119: 408-416.

Tran TT, Srivareerat M, Alkadhi KA (2010). Chronic psychosocial stress triggers cognitive impairment in a novel at-risk model of Alzheimer's disease. Neurobiol Dis 37: 756-763.

Tran TT, Srivareerat M, Alkadhi KA (2011b). Chronic psychosocial stress accelerates impairment of long-term memory and latephase long-term potentiation in an at-risk model of Alzheimer's disease. Hippocampus 21: 724-732.

Walker JJ, Terry JR, Tsaneva-Atanasova K, Armstrong SP, McArdle CA, Lightman SL (2010). Encoding and decoding mechanisms of pulsatile hormone secretion. J Neuroendocrinol 22: 1226-1238.

Wei Q, Hebda-Bauer EK, Pletsch A, Luo J, Hoversten MT, Osetek AJ et al (2007). Overexpressing the glucocorticoid receptor in forebrain causes an aging-like neuroendocrine phenotype and mild cognitive dysfunction. J Neurosci 27: 8836-8844.

Weiner MF, Vobach S, Olsson K, Svetlik D, Risser RC (1997). Cortisol secretion and Alzheimer's disease progression. Biol Psychiatry 42: 1030-1038.

Wilson RS, Evans DA, Bienias JL, Mendes de Leon CF, Schneider JA, Bennett DA (2003). Proneness to psychological distress is associated with risk of Alzheimer's disease. Neurology 61: 1479-1485.

Wilson RS, Schneider JA, Boyle PA, Arnold SE, Tang Y, Bennett DA (2007). Chronic distress and incidence of mild cognitive impairment. Neurology 68: 2085-2092.

Wolfer DP, Crusio WE, Lipp HP (2002). Knockout mice: simple solutions to the problems of genetic background and flanking genes. Trends Neurosci 25: 336-340.

Supplementary Information accompanies the paper on the Neuropsychopharmacology website (http://www.nature.com/npp) 